\title{
Measurement Characteristics of a Concept Classification Exam Using Multiple Case Examples: A Rasch Analysis
}

Nicholas B. Jennings, MPH, DrPH Candidate

College of Public Health

University of Arizona

Tucson, Arizona 85721

nbjennings@email.arizona.edu

Marion K. Slack, Ph.D. [corresponding author]

Professor

1295 N. Martin

PO Box 210202

College of Pharmacy

University of Arizona

Tucson, AZ 85721-0202

Phone: 520.626.1099

slack@pharmacy.arizona.edu

Lea E. Mollon, Pharm.D.

College of Pharmacy

University of Arizona

Tucson, Arizona 85721

mollon@pharmacy.arizona.edu

Terri L. Warholak, Ph.D.

College of Pharmacy

University of Arizona

Tucson, Arizona 85721

warholak@pharmacy.arizona.edu

Abstrac Objective: To determine if an exam using multiple case examples to test research design concepts measured only one cognitive skill, concept classification, and to determine if item difficulty varied according to the research design used for the case example. Methods: The exam consisted of 50 multiple choice items associated with five example abstracts: a randomized controlled trial, pretestposttest, crossover, retrospective cohort, and descriptive designs. A Rasch analysis was conducted to determine dimensionality (i.e. measured a single skill). Items were stratified by design to explore the relationship between item difficulty and study design. Overall difficulty was assessed using an itemperson map. Results: The exam was administered to 101 students; the mean was $88.4 \%$ (mean score $=$ 44.2; SD = 3.5). The Rasch analysis indicated the exam primarily measured one cognitive skill, presumably concept classification. Overall, the exam was easy, demonstrating that most students had mastered the material. The stratified analysis indicated that overall no one research design was more difficult than other designs, however, the type of research design and item topic interacted so that an 
easy item for one design could be difficult when associated with a different study design. Conclusions: That most students did well indicates that the exam functioned as a mastery exam rather than as an exam for ranking students by ability. That item topic interacted with study design to affect item difficulty, indicates that items on the same topic are needed to test basic design concepts across study designs.

Key Words: concepts, multiple choice exam, Rasch analysis, research design The authors have nothing to disclose 
Highlights:

- We conducted a Rasch analysis of a concept classification exam in research design.

- The exam measured one construct-concept classification of research designs.

- Exam items ranged in difficulty from very easy to slightly difficult.

- The exam functioned like a mastery exam; $98 \%$ of students passed the exam.

- Items interacted with research design type resulting in varying item difficulties. 
3

4

5

(n)

0

\section{Measurement Characteristics of a Concept Classification Exam Using Multiple Case Examples: A} Rasch Analysis

Abstract Objective: To determine if an exam using multiple case examples to test research design concepts measured only one cognitive skill, concept classification, and to determine if item difficulty varied according to the research design used for the case example. Methods: The exam consisted of 50 multiple choice items associated with five example abstracts: a randomized controlled trial, pretestposttest, crossover, retrospective cohort, and descriptive designs. A Rasch analysis was conducted to determine dimensionality (i.e. measured a single skill). Items were stratified by design to explore the relationship between item difficulty and study design. Overall difficulty was assessed using an itemperson map. Results: The exam was administered to 101 students; the mean was $88.4 \%$ (mean score = 44.2; SD = 3.5). The Rasch analysis indicated the exam primarily measured one cognitive skill, presumably concept classification. Overall, the exam was easy, demonstrating that most students had mastered the material. The stratified analysis indicated that overall no one research design was more difficult than other designs, however, the type of research design and item topic interacted so that an easy item for one design could be difficult when associated with a different study design. Conclusions: That most students did well indicates that the exam functioned as a mastery exam rather than as an exam for ranking students by ability. That item topic interacted with study design to affect item difficulty, indicates that items on the same topic are needed to test basic design concepts across study designs.

Key Words: concepts, multiple choice exam, Rasch analysis, research design

The authors have nothing to disclose 


\section{Introduction}

Cases or examples are often used for testing students on clinical or scientific skills in the health sciences. The student is presented with a clinical case then asked, for example, to identify an appropriate diagnosis for the patient, or an appropriate drug to treat the disease. If the example is an abstract of a research report, the student is asked to identify the type of research design or identify an appropriate statistical test for the reported findings. The cases represent examples from categories related to the topic under consideration. For instance, the patient could be an example of a patient with Type 2 diabetes who is being treated with metformin or the example could be an abstract from a randomized controlled trial (RCT) of metformin in the treatment of Type 2 diabetes. In both cases, students need classification skills to correctly categorize (diagnose) the patient as having Type 2 diabetes, categorize metformin as an appropriate therapy or categorize the design of the example research as that of an $\mathrm{RCT}$.

Concept classification theory as applied to instruction and testing has been described by Merrill and Tennyson. ${ }^{1}$ We have applied their approach to the development of instruction and testing of research design concepts. The research design course was required for students in the second year of the four year doctor of pharmacy program. The goal of the course was for students to be able to recognize the type of research design used in an example study and to be able to identify the study characteristics and the validity issues associated with each type of study design. The basic instructional strategy was to present an example study representing a specific design (e.g. a RCT), identify the pertinent characteristics (e.g. uses random assignment to groups), identify the type of statistical test used (e.g. ttest or Chi square), and identify the primary threat to internal validity for that research design (e.g. for a $\mathrm{RCT}$, the primary threat is experimental mortality). Exams consisted of abstracts for five different study designs with accompanying multiple choice questions asking students to identify the specific research design, identify characteristics of the study, including the type of statistical test used, and identify the primary threat to internal validity. Practice homeworks similar to the exam were provided throughout the instructional unit, however, all examples on the exam were new to the student.

Students generally do well on the exam; the class average ranges from 82 to 88 percent correct. However, we knew little about the measurement characteristics of the exam. We did not know if the use 
1 of multiple case examples might result in an exam that measured several different cognitive skills. It seemed that a student might use concept classification for one example and a different cognitive skill, for example, recall, for a different case example. We did not know whether we could assume that if a student correctly responded to an item on a specific topic (e.g. identify the independent variable) for one case example that they could respond correctly to the same question for a different case example. We did not know if items related to some topics were more difficult than items related to other topics. We assumed that the exam was at the appropriate level of difficulty but we had no evidence to support the assumption other than that the students seemed to do well overall. Finally, we had no information on reliability, guessing, or if there were problems with item response options. Therefore, we undertook a Rasch analysis to provide some insight into these questions.

Rasch Analysis

Rasch analysis assesses the performance of persons on a test and the performance of items composing the test based on a specific response model. The model posits that items range from easy to difficult with a transition zone between the two extremes in which a person may respond correctly to some items and incorrectly to others. The model also posits that persons range in ability from low to high; a person with high ability would have a higher probability of answering any item correctly than a person of lower ability and the pattern of their responses would be such that easier items were answered correctly with progressively less likelihood that they would answer more difficult items correctly. This pattern is unidimensional indicating that the items are measuring only one cognitive skill. The item difficulty pattern for specific exams can be examined statistically to determine if the response pattern is unidimensional. ${ }^{2}$

The first step in Rasch analysis is to estimate item difficulty and person ability. Item difficulty is estimated from the odds that a random person would respond correctly to an item; items that nearly everyone answers correctly are easy and items that few persons answer correctly are difficult. Person ability is estimated from the odds that a specific person would respond correctly to any item; a person who has a high odds of responding correctly is considered to have higher ability, a person with a low odds is considered to have lower ability. ${ }^{2}$

By transforming item difficulty and person ability estimates onto a logit scale (a logit is the log of the odds ratio), item difficulty and person ability are on the same equal interval scale allowing direct 
comparison of person ability and item difficulty. Usually an item-person graph is constructed where person ability is matched to item difficulty; typically the match is where the person has $50 \%$ probability of responding correctly to an item of specific difficulty. The person will have less than $50 \%$ probability of responding correctly to more difficult items (higher on the logit scale) and a greater than $50 \%$ probability of responding correctly to easier items (lower on the logit scale). ${ }^{2}$

The second step in a Rasch analysis is to statistically estimate the fit of the observed data for both items and persons to the Rasch model. Two types of statistics are used, infit and outfit. Infit statistics are the weighted mean difference in the observed and expected probabilities of responding correctly (MNSQ). MNSQs of around 1 indicate that there is no difference in the expected and observed responses beyond that due to random variation. Acceptable values for infit MNSQs for classroom exams are 0.75 to $1.3 .^{2-3}$ Outfit statistics are calculated from the sum of squared standardized residuals (unweighted) and are used to identify item and person outliers; that is, responses that are much more variable than expected based on the Rasch model. Infit and outfit statistics also are reported as standardized scores (Zstd) to provide another perspective on the fit of the observed scores to Rasch pattern by standardizing the MNSQ'S based on sample size. Generally, Zstd scores between -2 and +2 are considered to indicate a reasonable fit to the Rasch model. ${ }^{2-3}$

Generally values for infit and outfit above the specified range indicate an excess of variability. For items, it can indicate that there is a problem with the item, for example, poor wording or poor response options. For persons, higher values can indicate that the respondent was careless, or exhibited lucky guessing. Low values for infit and outfit indicate that variability in responses is less than expected by the Rasch model. For items, the low values could result from dependence between items or from responses conforming to a deterministic pattern. Low values for persons on outfit statistics could indicate that the person has special knowledge.

The Rasch analysis procedure also produces reliability statistics for both items and persons that range from 0 to 1 . Values near 1 for persons indicate that the ability order of persons could be replicated if they were retested on similar items. For items, values near 1 indicate that the order of item difficulty would be similar for a second exam with similar items. 
The purpose of this Rasch analysis was first to determine if an exam constructed to test research design concepts using multiple (5) case examples was unidimensional; that is, whether it measured primarily concept classification skill or multiple cognitive skills. Second, determine that if students responding correctly to an item on a specific topic (e.g. identify the independent variable) also respond correctly to a similar item for a different case example. Third, determine if items on some topics are more difficult than items on other topics. Fourth, make a judgement about whether the exam was at the appropriate level of difficulty. Finally, determine if any items had problems with poor wording or poor response options.

\section{Methods}

Study Design: This evaluation used a quality assurance approach to describe the measurement characteristics of a classroom exam used to test pharmacy students on research design concepts; that is, the classroom instruction was the usual instruction and the exam was part of the class requirements. The course was developed to teach the research design concepts required to interpret and evaluate health sciences literature and was a required course for students in the second year of the four-year professional pharmacy program. All exams that were completed during the regularly scheduled exam period were included in the analysis.

Instruments: The exam contained 50 multiple choice or true/false questions specifically developed to address the concepts of research design presented in the course. There were five abstracts of example research designs: RCT, descriptive, retrospective cohort, crossover, and pretest/posttest designs with eight to 12 questions associated with each design. Because the students were novices, that is, they had had little exposure to clinical literature and to study design, the study described in the abstract needed to be relatively simple, that is, involve one or two research questions, use relatively simple statistics for the main outcome variables, and generally not be too complex. Additionally, the abstract needed to be clearly written so that students would not have difficulty identifying the study characteristics.

The multiple choice and true/false items for testing concept classification were constructed using the method of Merrill and Tennyson. ${ }^{1}$ Two primary strategies were used to construct the items for the exam. In one strategy, the concept name was used in the item stem (e.g. The independent variable for this study is:) and specific examples of variables from the study were used as the response options (e.g. drug 
treatment, age, blood pressure, and gender). In the second strategy, a specific example of the concept from the case example was used in the item stem (e.g. To compare the mean VAS score for pain, the authors would have used:), then concept category names were used as the response options (e.g. t-test for dependent groups, t-test for independent groups, ANOVA for dependent groups, or Chi square). An example true/false item consisted of the statement: 'This study used parallel groups' which required the student to correctly categorize the example study. The exam also contained four recall items. Concept items can be distinguished from recall items by the necessity of referring to the case example to respond, for example, the recall item 'Which of the following best describes interval level measurement?' with response options of 'named categories, named categories in order, named categories in order with equal spaces between them' does not require students to refer to the case example to respond, rather, they need to recall the definition of the concept 'interval level measurement'.

\section{Data Collection Procedures}

The paper and pencil exam was administered during a regularly scheduled class. Students were allowed 90 minutes to complete the exam and they marked their responses on paper forms. The exams were graded electronically using a bubble scanner. Individual responses to each question were captured in an electronic file and exported to Excel ${ }^{\odot}$. The data set was de-identified by the instructor at the onset, thus this study does not meet the federal definition of human subjects research. The Rasch analysis was

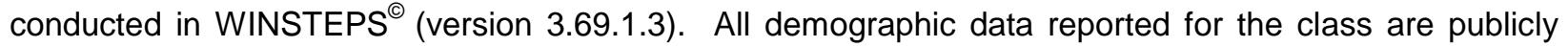
available through the College of Pharmacy.

\section{Data Analysis}

A Rasch analysis for dichotomous data (items scored correct or incorrect) was conducted. Infit and outfit statistics were reported as MNSQ's (a range of 0.7 to 1.3 was considered a fit) and Zstd's (a range of -2 to +2 was considered a fit) were used to determine if the response pattern for items and persons (students) was unidimensional. To determine if items associated with some types of study design were more difficult than others and to explore the relationship between item difficulty and specific study designs, an item difficulty map stratified by study design ${ }^{4}$ was constructed to enable visual inspection of item difficulty and to compare item difficulty between types of design. An item-person map was created to assess overall exam difficulty, and to identify items that were at the same level of difficulty 
1 (psychometrically redundant items). Rasch person and item reliability coefficients were calculated to

2 assess test reliability. The a priori alpha level was 0.05 .

3 Results

4 The class taking the exam consisted of 101 students, $34 \%$ male, in the second year of the 5 professional pharmacy curriculum. The average age of students was 23 . Fifty four percent of the class

6 had a bachelor's degree prior to admission to the professional program. The average student GPA at the $7 \quad$ time of admission was 3.63 .

The exam was administered to all 101 students during the scheduled exam period. The exam 9 contained 50 items and the exams were collected after 90 minutes. The mean number of correct items 10 was $44.2(88.4 \%)$, with a standard deviation of 3.5 . Three students achieved perfect scores (50/50, 11 100\%). The lowest score on the exam was $64 \%$ (32/50; 2 students).

12 The overall item statistics and maximum and minimum infit and outfit statistics from the Rasch 13 analysis, used to determine if there were problems with specific items, for each study design are shown in 14 Table 1. All MNSQ (desired range $=+0.7$ to +1.3 ) and Zstd (desired range $=-2.0$ to +2.0 ) statistics for 15 infit were within range ( 0.89 to 1.14 for MNSQ \& -1.0 to 1.1 for Zstd) indicating that the item difficulty 16 pattern was consistent with that expected if the exam were unidimensional. The MNSQ values for outfit 17 exceeded the maximum for the crossover design (MNSQ = 1.58) and the descriptive design (MNSQ = 18 1.97) and were below the minimum for the crossover design (MNSQ $=0.51$ ), the retrospective cohort 19 design $(\mathrm{MNSQ}=0.25)$, the pre-post design $(\mathrm{MNSQ}=0.20)$ and overall $(\mathrm{MNSQ}=0.20)$. The Zstd values 20 for outfit were within range (maximum observed $=2.0$, minimum $=-1.1$ ). Thus most of the evidence 21 indicates that the items were unidimensional and independent.

22 The item reliability was moderately high at 0.83 indicating that the order of item difficulty would likely 23 be similar if the exam were administered to similar group of students. The item separation index was 2.21 24 indicating that item difficulty ranks would be consistent if the exam were administered again.

25 The summary statistics for the entire group of students, used to determine if there was evidence of 26 carelessness, miscoding, lucky guessing, or special knowledge, are shown in Table 2. The MNSQ infit 27 statistics were all within range (observed maximum $=1.14 \&$ minimum $=0.76$ ) as were the Zstd infit 28 statistics (observed maximum $=0.4 \&$ minimum $=-1.1$ ) indicating that the pattern of student responses is 
consistent with the expected Rasch pattern. The outfit MNSQ statistics were somewhat out of range (minimum $=0.25$ and maximum $=2.35)$ however the Zstd statistics were within range $($ minimum $=-1.2 \&$ maximum $=+1.2)$. Hence, overall the fit statistics indicate that the observed pattern of response was a fairly good fit to the Rasch model and that there was no evidence of carelessness, miscoding, lucky guessing or special knowledge. However, student reliability was somewhat low (0.54) indicating that the order of student abilities may not be maintained with a similar group of students. The student separation index (1.01) indicates that ranking the ability of one student relative to another consistently was limited.

The item-person map delineating the relationship between student ability and item difficulty is shown Figure 1. The left side of the figure shows students ranked by level of performance on the exam with high performers at the top; three persons obtained perfect scores. The right side of the figure shows the exam items ranked by difficulty; the most difficult items are at the top. The logit scale for ranking both persons and test items is on the left of the figure. The shift of persons above the range of items by about 3 logits indicates that the exam was relatively easy; half the class had a greater than $50 \%$ chance of answering all items on the exam correctly. The shift of items below the person range indicates that all persons had a greater than $50 \%$ chance of answering the items below +0.7 logits correctly. There were not meaningful gaps between the items on level of difficulty except for the easiest items where there is about a one logit gap between the easiest item and the next two easiest items. The gap between the top of the item graph and the top of the person graph, indicates that there were no items that would differentiate between the students with the greatest ability.

Both persons and items were clumped in the middle of their respective distributions (see Figure 1). The clumping of persons indicates that, based on the scores of this exam, it would be difficult to distinguish between persons in the middle of the distribution which is reflected in the relatively low reliability index (0.54) shown in Table 1 . The clumping of items can indicate that some items are redundant relative to their difficulty; for example, the eight items located in a row at about -1 logit are equally difficult; so many items at the same level of difficulty may not be needed.

The difficulty of the items by the type of study design used in specific case examples (RCT, descriptive, retrospective cohort, crossover, \& pre-post) is shown in Figure 2. The logit scale is horizontal with the more difficult items on the right and the easier items on the left. In general, all types of study 
designs had items ranging from easy to difficult and the range of difficulty was similar indicating that, overall, the items associated with one design were not easier or more difficult than those associated with other designs. The possibly redundant items identified in Figure 1 are displayed in this graph as a column between -1 and -0.5 logits; at least one item is shown for each type of study design indicating that similar difficulty did not result from all items involving the same study design. Examination of the item topics for the eight items showed that the items involved six different topics. The topics include purpose of the study (2 items), comparison groups (2 items), and one item each on eligibility criteria, statistics, internal validity, and results. Hence the items were redundant relative to difficulty but not redundant relative to topic or relative to type of study design.

Further exploration of the association of item topic with difficulty level and type of study design, indicates that there may be an interaction between item topic and type of research design. For example, an item related to study purpose was included for each of four different study designs. The purpose item for the RCT was the easiest item on the exam (located at -3 logits), however, the purpose item associated with the retrospective cohort study was moderately difficult (located at +1 logits). In a similar manner, the most difficult item on the exam was an item on generalizability (+2.5 logits); two other generalizability items were much easier, one was located at about +0.3 logits and one was located at about -1 logit. The only item topic that overall seemed more difficult was statistics. Two of the five most difficult items involved statistics and the easiest statistical item located at about -0.6 logits was substantially more difficult than the easiest item on the exam (located at -3 logits).

\section{Discussion}

Important information about the function of the exam was obtained from the Rasch analysis. First, the analysis showed that the exam was unidimensional indicating that it tested primarily one cognitive skill, presumably concept classification skill, even though the exam consisted of multiple case examples representing different research designs. Second, item difficulty varied by study design, that is, an item that was easy when associated with one study design was difficult when associated with a different study design. In general, item difficulty was not associated with item topic, however, items related to statitistics did seem more difficult than other items. Third, the exam functioned like a mastery exam, that is, it demonstrated that most students had the ability to respond correctly to most of the items. Further, the 
exam did not differentiate between students of high ability (it could not rank one student as having more or less ability than a second student). Thus one could say that most students did well on the exam but one could not rank students relative to one another. Finally, there did not seem to be problems with the items on the exam. There was no evidence of poor wording, poor response options or evidence of lucky guessing, carelessness, miscoding, or special knowledge.

Jackson et al. ${ }^{3}$ conducted a Rasch analysis on an exam testing knowledge related to research design. Their exam consisted of one abstract for a research study with 25 multiple choice items. The example abstract was difficult; the study could have used a pre-post design with dependent groups (denoted as pre-experimental) or a before-after design with independent groups; the information in the abstract was not clear. Of note, is that the students taking the exam had not encountered a before-after design but had encountered a pre-post design. The difficulty of the one item on study design was about 2 logits above the highest scoring student on the person-item graph. The infit and misfit statistics indicated that the items related to this single case example formed a unidimensional scale.

Howard ${ }^{4}$ conducted a Rasch analysis of an exam testing the clinical knowledge of nurses on six disease states (heart failure, myocardial ischemia, conduction disturbances, structural defects, blood vessel disruption, and shock). The Rasch analysis of this 42 item exam showed that the items associated with shock and blood vessel disruption were somewhat easier than items for the other disease states. The person-item graph also showed that more than half the items were located below persons, indicating that students had attained a relatively high level of competence. No fit statistics were provided, therefore, the item and person fit to the Rasch model could not be evaluated.

\section{Implications}

The finding with the greatest implications for exam development was the finding that the difficulty of an item can vary depending on which research design it is associated with. For instance, one cannot assume that because students correctly identified the independent variable for an example RCT study that they can correctly identify the independent variable for a study using a pre-post design. Thus to create an exam that assesses level of competence, items on the same topic are needed for different case examples. 
The second finding with implications for exam development was the finding that the exam functioned like a mastery exam and that most students demonstrated a relatively high level of competence. In the context of a professional program, one wants to be able to demonstrate that all students have attained a relatively high level of competence. That one student cannot be ranked higher than another is relatively unimportant. Hence, even though the exam could be considered easy, it documents the competence of the students in the class. In contrast, an exam that reliably ranked students does not necessarily indicate if the highest performing student is competent; if that student had little chance of responding correctly to any of the items on the exam, then they could hardly be considered competent.

A third finding with implications for exam development was the finding that item reliability was

11 that there did not seem to be any problems with specific items. This finding is consistent with the notion 12 that using the method of item construction developed by Merrill and Tennyson ${ }^{1}$ for testing concept classification skills results in valid test items.

Another finding with implications for teaching in the research design course was the finding that items related to statistics seemed to be more difficult than items on other topics and that difficulty varied depending on which research design was used in the example. Perhaps more class time needs to be devoted to reviewing statistics or different instructional strategies need to be considered. Additionally, the variability in item difficulty between study designs indicates that design topics (e.g. identify the independent variable) need to be taught and tested for each research design.

The three primary factors affecting performance on the exam were probably student ability, including 22 study skills, instructional methods, and English as a second language. Because admission to the 23 professional pharmacy program is highly competitive and occurs after at least two years of prerequisite courses, all students in the class were assumed to have the basic ability and the basic study skills to do 25 well in the class. Thus student ability did not seem to be an issue.

The second factor related to student performance could be the instructional strategy used in the

27 class. The instructional strategy also involved the presentation of examples and identification of the 28 characteristics of each study design as well as the interpretation of information provided in study reports 
1 and abstracts. The instructional strategy was adapted from the strategy recommended by Merrill and

2 Tennyson. ${ }^{1}$ The instruction consisted of the same content as was tested on the exam; for instance, as

3 each example study design was considered, the identification of the independent variable associated with

4 the design was discussed. The same content was included in the homework; the major difference

5 between the class content, the homework, and the exam, was the use of new examples in the exam. If

6 students attended class and completed homework assignments, they should have had a reasonable

7 amount of skill in categorizing study designs and identifying study characteristics by the time they took the

8 exam.

9 A third factor that could have affected performance was English as a second language. Informal 10 observations by the instructors indicate that students who speak English as a second language seem to

11 have more difficulty with the course. Research design concepts and statistical concepts are very 12 abstract, hence, a student who has English skills that are even slightly limited may have difficulty 13 understanding the concepts. Hawkins ${ }^{5}$ also speculated that reading comprehension of English as a 14 second language could affect students' ability to interpret the item content.

15 Theory

16 Concept theory as operationalized by Merril and Tennyson ${ }^{1}$ appears to provide a useful framework 17 for developing items related to research design that test concept classification skills. The use of the 18 theory resulted in an exam that was unidimensional; an essential component of test validity as noted by 19 Bezruczko. ${ }^{6}$ Also, if the exam is unidimensional, then it becomes possible to isolate other factors that 20 affect item difficulty, for instance, to identify the interaction of the item topic with the study design resulting 21 in easier or more difficult items.

22 The findings from this evaluation are also consistent with the contention of Norman, Young, and Lee ${ }^{7}$ 23 that diagnostic reasoning (i.e. being able to correctly categorize a person as having one disease and not 24 another) is non-analytic reasoning. They note that learning requires multiple examples as were provided 25 in this research design class. They also discuss the importance of similarity to previously encountered 26 examples and the use of hypothesis testing. Both observations fit with the findings of this study. 27 Students could have compared the new examples on the exam to one or more examples encountered 
earlier. Students also could have used hypothesis testing to establish that an example abstract represented one design rather than another.

Limitations: There several limitations to this evaluation. Because the evaluation involved an exam administered as part of regular class work and the setting was not carefully controlled, there could be aspects of the setting that contributed to student performance on the exam that were not controlled. Also, because there was only one example of each study design, study design was completely confounded with the specific case example. Hence differences observed in the difficulty of item topics could have been influenced by the specific example used. Use of case examples that were relatively well written and relatively simple should have reduced the amount of confounding. Additionally, the study involved one exam on one content area administered to a single group of students at one institution, consequently generalizeability to exams involving other content areas or other groups of students is unknown.

\section{Conclusions}

The analysis indicates that the exam based on multiple case examples was unidimensional, that is, measured one cognitive skill, presumably concept classification skill. However, item topic interacted with type of study design to affect item difficulty indicating that items on the same topic are needed to test basic design concepts across different study designs even though their difficulty level may be similar. Also, the exam appeared relatively easy indicating that students could be identified as competent but could not be reliably ranked by ability. Finally, exam items developed using concept classification theory were reliable and had no problems with poor wording or poor response options.

0

1

2

3

4

5

6

7

8




\section{$1 \quad$ References}

1. Merrill MD, Tennyson RD. Teaching Concepts: An Instructional Design Guide. 1977. Englewood Cliffs: Educational Technology Publications.

2. Bond TG, Fox CM. Applying the Rasch model: Fundamental measurement in the human sciences. 2007. New York: Routledge.

3. Jackson TR, Draugalis JR, Slack MK, Zachry WM. Validation of authentic performance assessment: A process suited for Rasch modeling. American Journal Pharmaceutical Education. 2002;66 (3): 233-243.

4. Howard EP. (1985). Applying the Rasch Model to test administration. Journal of Nursing Education. $1985 ; 24: 340-343$.

5. Hawkins KW. Use of the Rasch model in communication educaton: An explanation and example application. Communication Education. 1987; 36 (2): 107-118.

6. Bezruczko N. Rasch Model Essentials. In Nikolaus Bezruczko (Ed.), Rasch Measurement in Health Sciences. 2005. Maple Grove: JAM Press.

7. Norman G, Young M, Brooks L. Non-Analytical Models of Clinical Reasoning: The Role of Experience. Medical Education. 2007; 41 (12): 1140-1145. 
Table 1: Rasch Infit and Outfit ${ }^{\text {a }}$ Statistics for Items by Type of Study Design

\begin{tabular}{|c|c|c|c|c|c|}
\hline \multirow[b]{2}{*}{ Research Design } & \multicolumn{2}{|c|}{ Infit Statistics } & \multicolumn{2}{|c|}{ Outfit Statistics } & \multirow[b]{2}{*}{ Question Content } \\
\hline & MNSQ $^{b}$ & Zstd $^{c}$ & MNSQ & Zstd & \\
\hline $\begin{array}{l}\mathrm{RCT}^{\mathrm{d}} \\
\text { Maximum value }\end{array}$ & 1.09 & 1.1 & 1.12 & 1.1 & Blinding \\
\hline Minimum value $^{f}$ & 0.94 & -0.1 & 0.77 & -0.4 & Internal validity \\
\hline Crossover $\quad$ Max & 1.14 & 0.8 & 1.58 & 2.0 & Comparison group \\
\hline Min & 0.89 & 0.0 & 0.51 & -0.5 & Study Conclusions \\
\hline $\begin{array}{l}\text { Pre-Posttest } \\
\text { Maximum value }\end{array}$ & 1.07 & 0.5 & 1.23 & 0.9 & Comparison groups \\
\hline Minimum value & 0.94 & 0.3 & 0.20 & -0.7 & Internal validity \\
\hline $\begin{array}{l}\text { Database } \\
\text { Maximum value }\end{array}$ & 1.05 & 0.3 & 1.01 & 0.2 & Comparison groups \\
\hline Minimum value & 0.90 & 0.1 & 0.25 & -0.9 & Timeframe \\
\hline $\begin{array}{l}\text { Descriptive } \\
\text { Maximum value }\end{array}$ & 1.06 & 0.3 & 1.97 & 1.4 & Statistical analysis \\
\hline Minimum value & 0.91 & -0.6 & 0.75 & -1.1 & Study Conclusions \\
\hline $\begin{array}{l}\text { Overall } \\
\text { Maximum value }\end{array}$ & 1.09 & 1.1 & 1.97 & 2.0 & (of all 50 questions) \\
\hline Minimum value & 0.89 & -1.0 & 0.2 & -1.1 & (of all 50 questions) \\
\hline
\end{tabular}

${ }^{\mathrm{a}}$ The Rasch model posits a response pattern consisting of three zones, an easy zone on one end, a difficult zone on the other end, and a transition zone between the two extremes. The infit statistics describe the deviation from the expected response pattern using weighted variances and indicates if there is more or less variability than expected from random variation. The outfit statistics use unweighted variance from the expected response pattern and are more sensitive to outliers, that is, items which contain substantial variation beyond random variation.

${ }^{\mathrm{b}} \mathrm{MNSQ}=$ mean square calculated from raw scores assesses the amount of variation from Rasch expected values; values between 0.7 and 1.3 indicate there is little variation beyond random variation.

${ }^{c}$ Zstd reports the variation from Rasch expected values based on standardized scores; values between -2 and +2 indicate there is little variation beyond random variation.

${ }^{\mathrm{d}} \mathrm{RCT}$ = randomized controlled trial

${ }^{\mathrm{e}}$ High values for MNSQ $(>1.3)$ or Zstd $(>+2)$ can indicate the presence of excess variability and that the item is not unidimensional (i.e. the item is testing more than one construct) or that the item is not independent of other items (Bold values indicate out-of-range values).

f Low values for MNSQ $(<0.7)$ or Zstd $(<-2)$ can indicate that there is less variation in item response patterns than would be expected from the Rasch model resulting from items that are similar or highly correlated. 
Table 2: Rasch Infit and Outfit ${ }^{\mathrm{a}}$ Statistics for Students

\begin{tabular}{|c|c|c|c|c|}
\hline \multirow[b]{2}{*}{ Students } & \multicolumn{2}{|c|}{ INFIT } & \multicolumn{2}{|c|}{ Outfit } \\
\hline & MNSQ $^{b}$ & Zstd $^{c}$ & MNSQ & Zstd \\
\hline Mean & 1 & 0.1 & 0.91 & 0.1 \\
\hline SD & 0.1 & 0.4 & 0.43 & 0.6 \\
\hline Maximum Values $^{d}$ & 1.14 & .4 & 2.35 & 1.2 \\
\hline Minimum Values & .76 & -1.1 & .25 & -1.2 \\
\hline
\end{tabular}

${ }^{b} \mathrm{MNSQ}=$ mean square calculated from raw scores assesses the amount of variation from Rasch expected values for persons (students); values between 0.7 and 1.3 indicate there is little variation beyond random variation.

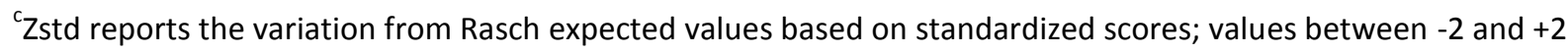
indicate there is little variation beyond random variation.

${ }^{\mathrm{d}}$ High values for MNSQ (>1.3) or Zstd ( > +2) can indicate the presence of excess variability, for example, the student has special knowledge or was guessing. (Bold values indicate out-of-range values).

${ }^{\mathrm{e}}$ Low values for MNSQ $(<0.7)$ or Zstd (<-2) can indicate that there is less variation in person (student) response patterns than would be expected from the Rasch model. 
Figure 1. Item Response Map

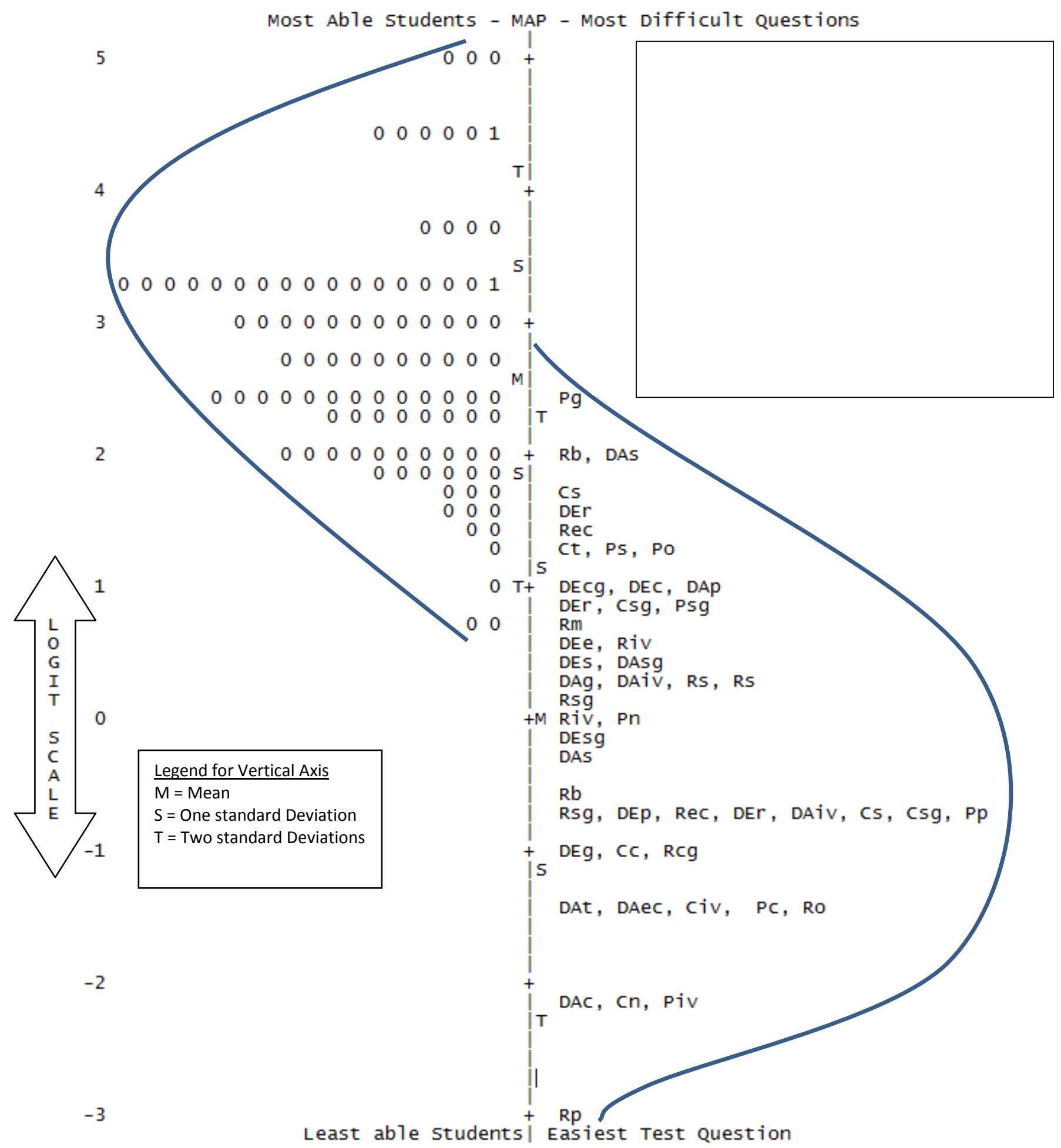


Figure 1. Scale values of test items by Study design and Concept

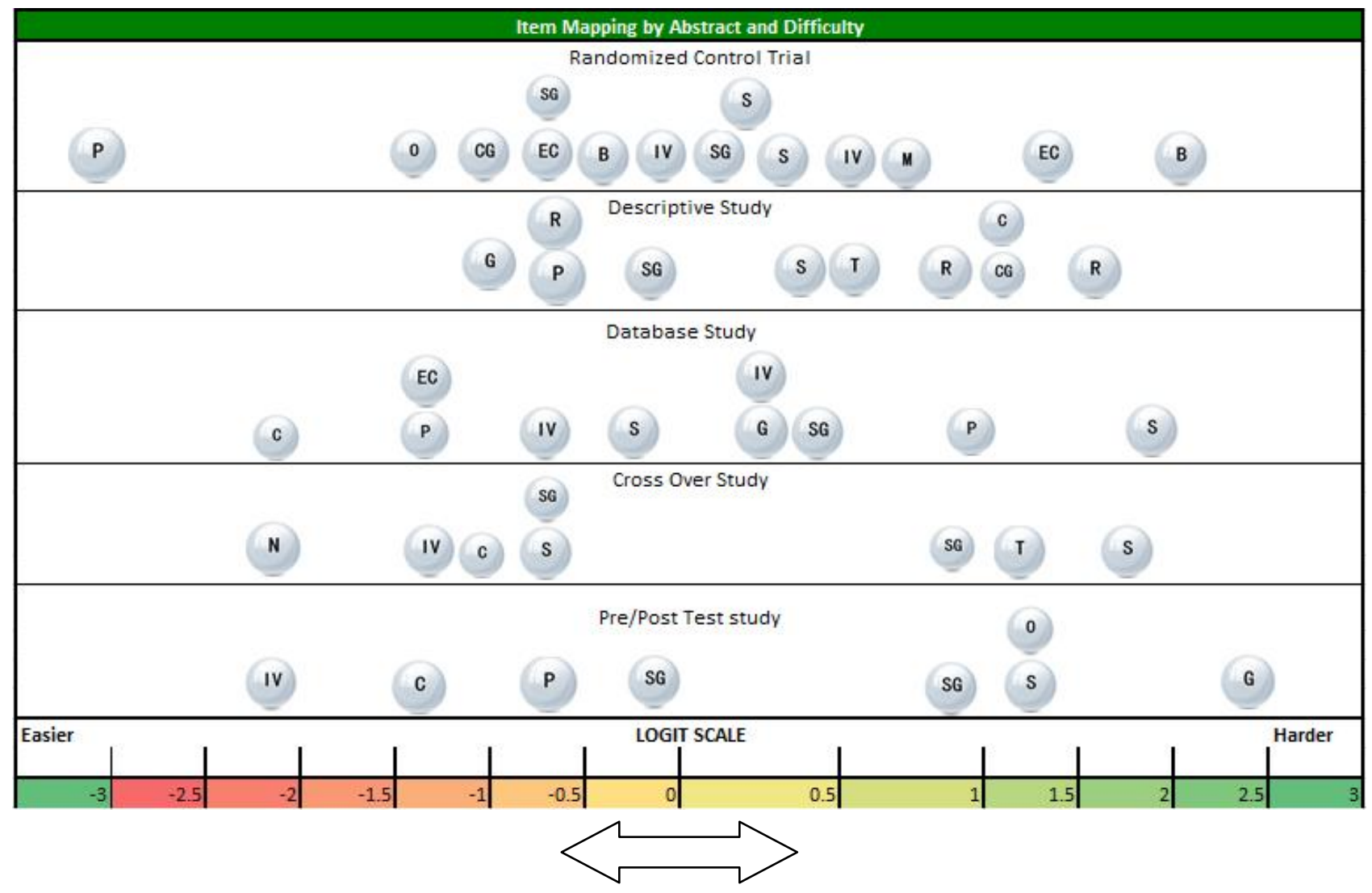

Question category abbreviations

$\begin{array}{ll}B=\text { Blinding } & C=\text { Conclusions } \\ G=\text { Generalizability } & I V=\text { Internal Validity } \\ P=\text { Purpose } & R=\text { Results } \\ T=\text { Time Frame } & \end{array}$

$\mathrm{B}=$ Blinding

$\mathrm{T}=$ Time Frame

$\begin{array}{ll}\text { CG }=\text { Comparison Groups } & \text { EC }=\text { Eligibility Criteria } \\ N=\text { Notation } & O=\text { Order (Parallel vs. Sequential Groups) } \\ S=\text { Statistical Tests } & \text { SG }=\text { Study Groups }\end{array}$

CG = Comparison Groups

\section{$\mathrm{S}=$ Statistical Tests $\quad \mathrm{SG}=$ Study Groups}

